\title{
Subject Index to Volume 28
}

Aciduria, $\mathrm{D}(+)$-glyceric, etiology and clinical consequences, 38

Adenosine deaminase deficiency, new enzyme replacement therapy, comparison, red cell transfusion, 127

Adipose tissue, role in glucose intolerance, obese offspring of hyperglycemic rats, 641

Adolescence

anthropometric measurements, genetic and environmental contributions, twin study, 1

sexually active females, papillomavirus infection prevalence, 507

$\beta$-Adrenergic receptor, concentration, cultured human fetal lung, 190

Age, erythropoietic response and, adult and infant monkeys, 567

Airway

electrical field stimulation, inhibitory and excitatory responses, fetal and adult pig, 69

obstruction

arousal and cardiopulmonary responses, carotid denervation effect, lamb, 374

ventilator for resistive unloading, rabbit, 79

pressure, during high-frequency oscillatory ventilation, normal rabbit model, 367

Allergy, cow's milk, local immune responses, 582

American Pediatric Society

Howland Award Presentation

comments of recipient, 415

introduction, 411

presidential address, 562

Amino acids, placental transfer, intrauterine growth retardation, guinea pig, 203

Aminophylline, effect on diaphragmatic contractility, neonatal piglet, 196

Amylase, breast milk, effects of prolonged lactation and maternal parity, 502

Angiotensionogen, gene expression, developmental regulation, sheep, 183

Anion, transport, sickle red blood cells, 587

Antibody, monoclonal, prevention and therapy, neonatal Escherichia coli infection, 667

Apnea, hypoxic, spontaneous recovery from, maturational adaptations, mice, 87

Apolipoprotein A-I, lipoproteins containing, insulin-dependent diabetes mellitus, 42

Asphyxia, brain superoxide anion generation during, newborn pig, 618

Atherosclerosis, accelerated, insulin-dependent diabetes mellitus, 42

Autoresuscitation, cardiorespiratory interactions, maturation, mice, 87

Bicarbonate, permeability, developmental changes, juxtamedullary proximal convoluted tubule, rabbit, 663

Bile, secretion of trace elements, glutathione role, rat, 339

Biotin

ileal transport, developmental maturation, rat, 266

labeling, red cells, preterm infants, 199
Blood-brain barrier, permeability, liver toxin effect, rabbit, 227

Blood flow

cerebral, extracorporeal membrane oxygenation effect, neonatal sheep, 50

diaphragmatic, patent ductus arteriosus effect, lamb, 437

during hypoxia, fetal hind limb, sheep, 94 placental, gestational changes, normal human fetus, 383

pulmonary, effect of systemic arterial pressure elevation, hypertensive piglet, 603

regional distribution, sleep effect, piglet, 218

venous, reversal with atrial tachycardia and hydrops, fetal sheep, 223

Blood spot, cholesterol screening, Australian neonates, 496

Body size, anthropometric measures, genetic analysis, twin study,

Bowel disease, inflammatory, intestinal $\gamma / \delta$ receptor-bearing $\mathrm{T}$ lymphocytes, 579

Brain

blood flow distribution, during tolazolineinduced hypotension, neonatal dog, 111

cerebral energy metabolism, during hypoxia, age dependence, dog, 54

childhood medulloblastoma, elevated cmyc expression, 63

electrocortical activity, analysis, fetal lamb, 106

metabolism, during tolazoline-induced hypotension, neonatal dog, 111

neurotransmitters, changes after thyroidectomy, fetal and neonatal lamb, 469

RNA levels, thyroid hormone receptor-related, rat, 622

superoxide anion generation, during asphyxia and reventilation, newborn pig, 618

vascular prostacyclin production, pressure ventilation effect, newborn pig, 609

Brainstem, auditory evoked response, gentamicin effect, term infant, 232

Breastfeeding

energy expenditure and deposition related to, comparison, formula-fed infants, 631

prolonged, breast-milk amylase activity and, 502

Breathing: see Respiration

Brent W. Brees/Dows Metabolic Research Symposium, introduction, 524

Calcitonin, serum concentrations, postnatal surge, infants of diabetic mothers, 493

Calcium

contractile response to, gall bladder smooth muscle, newborn and adult guinea pigs, 336

transport, intestinal Golgi vesicles, hypertensive rat, 591

Calmidazolium, myofibril stimulation by, rat heart, 46

Cardiomyopathy

carnitine-responsive, skin fibroblast carnitine uptake and, 247 deficiency of long-chain 3-hydroxyacylcoenzyme A dehydrogenase and, 657

Carnitine

deficiency, impaired skin fibroblast carnitine uptake in, 247

ester, excretion, parenteral nutrition patients, 158

L-Carnitine, pretreatment, before lethal ammonium acetate, mice, 256

Carotid, denervation, effect on arousal response to upper airway obstruction, lamb, 374

Celiac disease, intestinal $\gamma / \delta$ receptor-bearing T lymphocytes, 572

Central nervous system, involvement, erythrophagocytic disorders of infancy, 116

Cerebral blood flow, regional, after hemorrhagic hypotension, developmental changes, lamb, 361

Cerebrospinal fluid, oxypurine concentrations, hypoxemic pig, 477

Cholesterol screening, dried blood spots, Australian neonates, 496

Circulatory arrest, hypothermic, physiologic and neuropathologic aspects, newborn dog, 354

Complement receptor, expression, neonatal and preterm neutrophils, 120

Cord blood, bactericidal activity, effect of murine hybridoma antibodies, 667

Cow's milk, allergy, immunopathogenic mechanisms, 582

Cytochrome c oxidase

deficiency, overview, 536

mammalian isoforms, human cytochrome c oxidase deficiency and, 529

Deoxyadenosine triphosphate, measurement, adenosine deaminase deficiency, 127

Deoxyribonucleic acid

mitochondrial

mutations, analysis and diagnosis, neuromuscular disease, 525

progressive increase, Kearns-Sayre syndrome, 131

Dexamethasone

effect on $\beta$-adrenergic receptor concentration, cultured human fetal lung, 190

tropoelastin synthesis and, rat pulmonary fibroblasts, 379

Diabetes

insulin-dependent

accelerated atherosclerotic process in, 42 lipoproteins containing apoA-I in, 42 maternal, neonatal hypocalcemia and, 493

Diaphragm

blood flow, effect of patent ductus arteriosus, lamb, 437

contractility, aminophylline effect, neonatal piglet, 196

Diet, neonatal macromolecular absorption and, neonatal rat, 31

Dimethyl sulfoxide, protective effect, psychosine cytotoxicity, newborn rat neural cells, 473

Dopamine, effect on superoxide anion pro- 
duction, neonatal polymorphonuclear leukocytes, 24

Editor's statement, Duplicate publication and related problems, 561

Embryo, heart, conduction system functions, rat, 425

Endotoxin, discrimination between low dietary zinc and exposure to, weaning rat, 332

Energy

expenditure, protein metabolism and, preterm infant, 147

expenditure/deposition, breast-fed and formula-fed infants, 631

Environment, body size influenced by, preadolescent twins, 1

Enzyme, replacement therapy, glycogenosis type II, rat heart perfusion model, 344

Epinephrine, $\beta$-receptor mediated responses, desensitization by ritodrine, fetal lamb, 388

Erythrophagocytic lymphohistiocytosis, familial, central nervous system involvement, 116

Erythropoietin, recombinant human, erythropoiectic response, age-related differences, monkey, 567

Escherichia coli, $\mathrm{K} 1$ strain, effect of murine hybridoma antibodies against, 667

Estrogen, low-dose, increased growth hormone production rate after, prepubertal girls with Turner's syndrome, 626

Ethanol, maternal ingestion, effect on fetal vitamin A, rat, 186

European Society for Pediatric Research, abstracts for the 1990 annual meeting, 277

Fatty acid, omega-3, retinal function and, very-low-birth-weight neonates, 485

Fetus

airway response, electrical field stimulation, pig, 69

electrocortical activity, patterns, identification and quantitation, lamb, 106

glucose utilization, effect of maternal uterine artery ligation, rat, 464

hepatic angiotensinogen gene expression, developmental regulation, sheep, 183

hypoxia, theophylline stimulation of breathing movements during, sheep, 83

intrauterine growth retardation, placental transfer of glucose and amino acids, guinea pig, 203

lactate metabolism, normal and growthretarded, 652

lung, $\beta$-adrenergic receptors and CAMP response increase during explant culture of, 190

placental blood flow, gestational changes, 383

Tetralogy of Fallot and pulmonary hypertension, rat, 429

thyroidectomy, brain transmitter changes after, lamb, 469

venous blood flow reversal, during atrial tachycardia and hydrops, sheep, 223

vitamin A levels, maternal ethanol ingestion and, rat, 186

Follicle stimulating hormone, monthly urinary secretory patterns, normal children and patients with sexual disorders, 405

Functional residual capacity, automated bedside measurement, mechanically ventilated children, 446, 451
Gallbladder, smooth muscle contractility, calcium response, newborn and adult guinea pigs, 336

Genetics, body size influenced by, preadolescent twins, 1

Gentamicin, effect on auditory brainstem evoked response, term infant, 232

Glomerular sclerosis, mechanism, maturing rat kidney, 270

Glucagon, hepatic glycogen degradation and, newborn rat, 646

Glucoamylase, role in glucose polymer hydrolysis, rat intestine, 166

Glucose

fetal tissue utilization, effect of maternal uterine ligation, rat, 464

metabolism

adipocytes, obese offspring of mild hyperglycemic rats, 641

during total parenteral nutrition, preterm infant, 153

placental transfer, intrauterine growth retardation, guinea pig, 203

polymer, hydrolysis and absorption, intestinal glucoamylase role, rat, 166

Glutathione, hepatobiliary transport, effect on bile secretion of trace elements, rat, 339

Glyceric acid, urinary excretion, clinical findings and long term effects, 38

Glycogenolysis, hepatic, hyperinsulinemia effect, newborn rat, 646

Glycogenosis, type II, enzyme replacement therapy, rat heart perfusion model, 344

Glycogen storage disease Ib, hexose uptake and transport, polymorphonuclear leukocytes, 19

Golgi vesicle, intestinal, calcium transport, hypertensive rat, 591

Gonadotropin, urinary excretion, age-related changes, 401

Gonadotropin-releasing hormone, sleep-associated changes, during sexual maturation, 213

Great vessel, anomalies, Tetralogy of Fallot and, fetal and neonatal rat, 429

Growth and development

angiotensinogen gene expression, developmental regulation, sheep, 183

autoresuscitation, maturation of cardiorespiratory interactions, mice, 87

bicarbonate permeability, juxtamedullary proximal convoluted tubules, rabbit, 663

biotin transport, rat ileum, 266

cerebral energy metabolism, during hypoxia, neonate and adult dogs, 54

gonadotropin excretion, age-related changes, 401

growth hormone secretion, developmental changes, neonatal monkey, 15

hypoxia effect, myocardial purines, immature and mature rabbit, 348

lung, diet-induced sodium deficiency and, young rat, 455

myofibrillar activity, differential effect, calmidazolium, neonatal and adult rat, 46

neurotransmitters in hypothyroidism, fetal and neonatal lamb, 469

placental blood flow, gestational changes, 383

pubertal, in chronic renal failure, 5

regional cerebral blood flow, after hemorrhagic hypotension, preterm, nearterm, and newborn lamb, 361

sexual maturation, sleep modulation of neuroendocrine function during, 213

short stature, pulsatile growth hormone release variations, 11

small intestine, oral insulin effect, neonatal miniature pig, 171

smooth muscle contractility, gallbladder, newborn and adult guinea pigs, 336

thyroid receptor RNA levels, developing rat brain, 622

tropoelastin synthesis, rat pulmonary fibroblasts, 379

Growth hormone

effect on nitrogen kinetics, short children, 394

low-dose estrogen effect on production rate of, prepubertal girls with Turner's syndrome, 626

pulsatile release, variations, healthy short prepubertal boys, 11

urinary output, small for gestational age infants, 209

Growth retardation

intrauterine, placental transfer of glucose and amino acids, guinea pig, 203

maternal-fetal lactate concentrations and, 652

Heart

conduction system functions, rat embryo, 425

filament activity, calmidazolium stimulation, developmental difference, rat, 46

hypertrophy, Tetralogy of Fallot and, fetal and neonatal rat, 429

Hematopoietic progenitors, cycling status, interleukin 6 effect, newborn and adult rats, 323

Heteroplasmy, clinical and molecular studies, chronic external ophthalmoplegia, 542

Hexose, uptake and transport, glycogen storage disease $\mathrm{Ib}, 19$

Hormone

secretion patterns, menstruating women with Turner syndrome, 514

see also specific name

Hydrops, venous blood flow reversal during, fetal sheep, 223

3-Hydroxyacyl-coenzyme A dehydrogenase, long-chain, deficiency, lethal myopathy and, 657

Hyperammonemia, L-carnitine as protective agent, mice, 256

Hypercapnia, cerebral perfusion during, role of brain vascular prostacyclin production, 609

Hyperinsulinemia, impaired hepatic glycogenolysis related to, newborn rat, 646

Hypertension

calcium transport and, intestinal Golgi, rat, 591

pulmonary, Tetralogy of Fallot and, fetal and neonatal rat, 429

right ventricular, systemic arterial pressure elevation during, piglet, 603

Hypocalcemia, neonatal, infants of diabetic mothers, 493

Hypotension

hemorrhagic, regional cerebral blood flow after, developmental changes, lamb, 361

tolazoline-induced, blood flow distribution and brain metabolism during, neonatal dog, 111

Hypothermia, circulatory arrest due to, physiologic and neuropathologic aspects, newborn dog, 354

Hypothyroidism, congenital, brain neuro- 
transmitters and, fetal and neonatal lamb, 469

Hypoxanthine

plasma, urine and cerebrospinal fluid concentrations, hypoxemic pig, 477

vitreous humor levels, changes during hypoxemia and post-mortem, pig, 482

Hypoxemia

oxypurine concentrations during

cerebrospinal fluid, plasma, urine, pig, 477

vitreous humor, pig, 482

Hypoxia

acute graded, oxygen consumption and blood flow during, fetal hind limb, sheep, 94

breathing movements during, theophylline stimulation, fetal sheep, 83

cerebral energy metabolism during, age dependence, neonate and adult dogs, 54

effect on myocardial purines, immature and mature rabbit heart, 348

intrauterine, identification of growth-retarded fetuses at risk for, 652

lleum, biotin transport, developmental maturation, rat, 266

Infant: see also neonate

auditory brainstem evoked response, gentamicin effect, 232

malnutrition, school achievement after early kwashiorkor or marasmus, 235

preterm

glucose metabolism during total parenteral nutrition, 153

red cell volume measurement, 199

relationship, protein metabolism and energy expenditure, 147

small for gestational age, urinary growth hormone and insulin-like growth factor I excretion, 209

Infant formula, early weaning to, macromolecular absorption and, neonatal rat, 31

Inferior vena cava, flow reversal, during atrial tachycardia and hydrops, fetal sheep, 223

Insufflation time, passive deflation time con-

Insulin stant and, juvenile rabbit lung, 75

hepatic glycogen degradation and, newborn rat, 646

oral, effect on small intestinal growth and development, neonatal miniature pig, 171

Insulin-like growth factor I, urinary output, small for gestational age infants, 209

Interferon- $\alpha$, responsiveness of natural killer cells to, infancy to early childhood, 316

Interleukin 2, responsiveness of natural killer cells to, infancy to early childhood, 316

Interleukin 6, effect on hemopoietic progenitors, newborn and adult rats, 323

Intestine, ornithine metabolism, development, mouse, 261

Kearns-Sayre syndrome, mutated mitochondrial DNA fraction, progressive increase, 131

Keto-Doxapram, pharmacodynamic effects, comparison, doxapram, neonatal lamb, 142

Kidney

chronic renal failure, pubertal growth in, 5 interstitial hydrostatic pressure, role, blunted natriuretic response to saline loading, piglet, 460

Krabbe's disease, psychosine cytotoxicity, rat neural cell cultures, newborn rat, 473

Kwashiorkor, during infancy, school achievement after, 235

Lactate, metabolism, normal and growth-retarded fetuses, 652

Lacticacidemia, respiratory chain defects, detection, skin fibroblast cultures, 549

Lactoferrin, structural homology, shared antigenic determinants, human, bovine, porcine, 176

Latin-American Society for Pediatric Research, abstracts of 27 th annual meeting, 556

Latin-American Society of Pediatric Endocrinology, abstracts of 3rd meeting, 419

Lesch-Nyhan syndrome, protein metabolism in, 240

Leukemia, acute lymphoblastic, recycling defect, natural killer cells, 572

Lipid, peroxidation, role, oxygen-induced lung injury, newborn lamb, 613

Liver

angiotensinogen gene expression, developmental regulation, sheep, 183

failure, serum toxins during, blood-barrier permeability and, 227

Lung

fetal, $\beta$-adrenergic receptors and cAMP response increase during explant culture of, 190

fibroblast, tropoelastin synthesis, developmental changes, rat, 379

hypoplasia, oligohydramnios-induced, vasopressin role, rat, 595

mechanics, juvenile rabbit, 75

normal and compensatory growth, dietinduced sodium deficiency and, young rat, 455

oxygen-induced injury, lipid peroxidation role, newborn lamb, 613

Luteinizing hormone, monthly urinary secretory patterns, normal children and patients with sexual disorders, 405

Lysyl oxidase, reduced activity, skin and aorta, Menkes' disease, 137

Malnutrition, early childhood, long-term effects, 235

Marasmus, during infancy, school achievement after, 235

Maturation, sexual, changes in hormone secretion during, 213

Mechanical ventilation: see Ventilation

Medulloblastoma, c-myc activation, possible mechanisms, 63

Menkes' disease, reduced lysyl oxidase activity, skin and aorta, 137

Menses, Turner syndrome patients with, hypothalamic-pituitary-ovarian function, 514

Messenger ribonucleic acid

c-myc, elevated levels, childhood medulloblastomas, 63

hepatic and renal angiotensinogen expression, fetal, newborn, and adult sheep, 183

Metabolism, inborn errors, deficiency of long-chain 3-hydroxyacyl-coenzyme A dehydrogenase, 657

Methylbutanoic acid, incorporation, cultured fibroblasts, suspected 3-oxothiolase deficiency, 518
Milk, lactoferrin, shared antigenic determinants, human, bovine, porcine, 176

Mucosa, permeability, oxidant-induced increases, neonatal piglet, 28

Muscle, skeletal, oxygen extraction in, lamb, 101

NADPH oxidase, membrane activity, cell size and, bovine neonatal neutrophils, 327

Natriuresis, volume expansion-induced, role of renal interstitial hydrostatic pressure, piglet, 460

Natural killer cell, cytotoxicity, development, infancy to early childhood, 316

Natural killer cells, recycling defect, acute lymphoblastic leukemia, 572

Neonate

apo A-I, apo B, and apo(a) levels, dried blood spots, Australian study, 496

asphyxia, brain superoxide anion generation during, pig, 618

cerebral blood flow, extracorporeal membrane oxygenation effect, sheep, 50

cycling status of hematopoiectic progenitors, interleukin 6 effect, rat, 323

diaphragmatic contractility, aminophylline effect, piglet, 196

growth hormone response to growth hormone-releasing hormone, longitudinal changes, monkey, 15

hypercapnia, increased ventilation pressure during, piglet, 609

hyperglycemic, impaired hepatic glycogenolysis, rat, 646

hypocalcemia, calcitonin role, infants of diabetic mothers, 493

hypotension, blood flow distribution and brain metabolism, neonatal dog, 111

hypothermic circulatory arrest, physiologic and neuropathologic aspects, dog, 354

hypoxia, cerebral energy metabolism during, dog, 54

jejunal macromolecular absorption, early weaning effect, rat, 31

mucosal permeability, oxidant-induced increases, piglet, 28

ornithine metabolism, development, mouse intestine, 261

oxygen toxicity, biochemical manifestations, newborn lamb, 613

polymorphonuclear leukocytes, respiratory burst, dopamine effect, 24

polymorphonuclear neutrophils, NADPH oxidase activity and cell size in, calf, 327

psychosine cytotoxicity, rat neural cell cultures, 473

red cell volume measurement, biotin labeling method, 199

respiration, comparison pharmacodynamic effects, keto-doxapram and doxapram, 142

reventilation, brain superoxide anion generation during, pig, 618

right ventricular hypertension, systemic arterial pressure elevation during, piglet, 603

small-for-gestational age, supranormal glucose requirements, rat, 464

small intestinal growth, oral insulin effect, neonatal miniature pig, 171

Tetralogy of Fallot and pulmonary hypertension, rat, 429

thyroidectomy, brain transmitter changes after, lamb, 469

thyroid receptor RNA levels, rat brain, 622 
very-low-birth-weight, retinal function, dietary omega- 3 fatty acid effect, 485

Neopterin, central nervous system, role in erythrophagocytic disorders, 116

Nephrectomy, subtotal, glomerular sclerosis after, maturing rat kidney, 270

Neuromuscular disease, mitochondrial DNA mutations and, polymerase chain reaction analysis, 525

Neutrophil

fetal, complement receptor expression, $\mathrm{Rh}$ disease, 120

polymorphonuclear, NADPH oxidase activity and cell size in, newborn calves, 327

Nitrogen

washout, bedside measurement of functional residual capacity, ventilated children, 446, 451

whole body kinetics, growth hormone treatment and, 394

Nutrition

parenteral, carnitine ester excretion, 158

total parenteral, glucose metabolism during, preterm infant, 153

Obesity

genetic and environmental contributions, preadolescent twins, 1

glucose intolerance and, rat adipocyte, 641

Ophthalmoplegia, chronic external, heteroplasmy, clinical and molecular studies, 542

Ornithine, metabolism, development, mouse intestine, 261

3-Oxothiolase, deficiency, measurement, cultured fibroblasts, 518

Oxygen

cerebral metabolism, extracorporeal membrane oxygenation effect, sheep, 50

consumption, hind limb, during hypoxia, fetal sheep, 94

extraction, lamb skeletal muscle, 101

toxicity, biochemical manifestations, newborn lamb, 613

Oxygenation, extracorporeal membrane, cerebral blood flow, neonatal sheep, 50

Papillomavirus, prevalence and risk factors, sexually active adolescent females, 507

Patent ductus arteriosus, effect on diaphragmatic blood flow and function, lamb, 437

Phenylketonuria, protein metabolism in, 240

Phorbol ester, protective effect, psychosine cytotoxicity, newborn rat neural cells, 473

Placenta, blood flow, gestational changes, normal human fetus, 383

Plasma, oxypurine concentrations, hypoxemic pig, 477

Polymerase chain reaction, analysis, mitochondrial DNA mutations associated with neuromuscular diseases, 525

Polymorphonuclear leukocyte

hexose uptake and transport in, glycogen storage disease $\mathrm{Ib}, 19$

respiratory burst, dopamine effect, neonate, 24

Pompe's disease, enzyme replacement therapy, rat heart perfusion model, 344

Pregnancy, ethanol ingestion during, effect on fetal vitamin A, rat, 186

Prematurity: see also Infant, preterm anemia of, erythropoietin treatment, adult and infant rhesus monkeys, 567

Pressure ventilation, brain vascular prostacyclin increased by, newborn pig, 609

Prostacyclin, pressure ventilation and production of, newborn pig, 609

Protein

metabolism

energy expenditure related to, preterm infant, 147

phenylketonuria and Lesch-Nyhan syndrome, 240

Proximal tubule, permeability, developmental changes, rabbit, 663

Psychosine, cytotoxicity, rat neural cell cultures, newborn rat, 473

Puberty, growth during, chronic renal failure patients, 5

Purine, myocardial, hypoxia effect, immature and mature rabbit, 348

Red blood cell

biotin labeling, preterm infants, 199

sickle, anion transport, 587

Renal failure: see Kidney

Resonant frequency, total respiratory system compliance calculated from, rabbit, 599

Respiration

during hypoxia, theophylline stimulation, fetal sheep, 83

spontaneous, resistive unloading during, ventilator technique for, rabbit, 79

Respiratory chain, defect, detection by skin fibroblast cultures, lacticacidemic patients, 549

Respiratory system, total compliance, calculation, rabbit, 599

Retina, effect of omega-3 fatty acids, verylow-birth-weight neonate, 485

Reventilation, brain superoxide anion generation during, newborn pig, 618

Ribonucleic acid, thyroid hormone receptor and, developing rat brain, 622

Rice, glucose polymer, hydrolysis, role of rat intestianl glucoamylase, 166

Ritodrine, prolonged treatment, effect on epinephrine response, fetal lamb, 388

Saline loading, blunted natriuretic response to, role of renal interstitial hydrostatic pressure, piglet, 460

School performance, after early childhood malnutrition, 235

Sexual behavior, papillomavirus infection and, adolescent female, 507

Sexual disorder, urinary LH and FSH secretion, monthly patterns, 405

Short stature, pulsatile growth hormone release variations, prepubertal boys, 11

Skin fibroblast, impaired carnitine uptake, in primary systemic carnitine deficiency, 247

Sleep

neuroendocrine function and, during sexual maturation, 213

regional blood flow distribution and, piglet, 218

upper airway obstruction during, carotid denervation effect, lamb, 374

Small intestine, macromolecular absorption, early weaning effect, rat, 31

Smooth muscle, calcium response, gallbladder, newborn and adult guinea pigs, 336

Society for Pediatric Research, Presidential Address 1990, 313
Sodium, deficiency, effect on normal and compensatory lung growth, young rat, 455

Superoxide, anion generation, during asphyxia/reventilation, newborn pig, 618

Superoxide anion, production by neonatal polymorphonuclear leukocytes, dopamine effect, 24

Tachycardia, atrial, venous blood flow reversal during, fetal sheep, 223

Tetralogy of Fallot, pulmonary hypertension and, fetal and neonatal rat, 429

Theophylline, respiratory responses to, during normoxia and hypoxia, fetal sheep, 83

Thyroidectomy, brain neurotransmitter changes after, fetal and neonatal lamb, 469

Tidal volume, passive deflation time constant and, juvenile rabbit lung, 75

$\mathrm{T}$ Lymphocyte, $\gamma / \delta$ receptor-bearing, celiac disease and inflammatory bowel disease, 579

Trace elements, bile secretion, glutathione role, rat, 339

Tropoelastin, synthesis, rat pulmonary fibroblasts, developmental changes, 379

Troponin I, developmental isoform transitions, myofibril stimulation by calmidazolium and, neonatal rat heart, 46

Turner's syndrome

low-dose estrogen therapy, increased growth hormone production rate after, prepubertal girls, 626

menstruating women with, hypothalamicpituitary-ovarian function, 514

Uric acid

plasma, urine and cerebrospinal fluid concentrations, hypoxemic pig, 477

vitreous humor levels, changes during hypoxemia and post-mortem, pig, 482

Urine, oxypurine concentrations, hypoxemic pig, 477

Uterine artery, ligation, maternal, fetal tissue glucose utilization and, rat, 464

Vasopressin, role, oligohydramnios-induced lung hypoplasia, rat, 595

Ventilation

high-frequency oscillatory, proximal, tracheal, alveolar pressures during, rabbit, 367

mechanical, bedside measurement of functional residual capacity, new automated technique, 446,451

Ventilator, negative resistance, applicability, airway obstruction, rabbit, 79

Vitamin A, metabolism, maternal ethanol ingestion and, fetal rat, 186

Vitreous humor, oxypurine concentrations, changes during hypoxemia and postmortem, pig, 482

Xanthine

plasma, urine and cerebrospinal fluid concentrations, hypoxemic pig, 477

vitreous humor levels, changes during hypoxemia and post-mortem, pig, 482

Zinc, low dietary status, discrimination, endotoxin exposure, weaning rat, 332 